\title{
Supplementary Information: \\ Mutations to R. sphaeroides Reaction \\ Center Perturb Energy Levels and Vibronic \\ Coupling but Not Observed Energy \\ Transfer Rates
}

Moira L. Flanagan ${ }^{1}$, Phillip D. Long ${ }^{1}$, Peter D. Dahlberg ${ }^{1}$, Brian S. Rolczynski ${ }^{2}$, Sara C. Massey ${ }^{2}$, and Gregory S. Engel ${ }^{2}$

${ }^{1}$ Graduate Program in the Biophysical Sciences, The James Franck Institute and The Institute for Biophysical Dynamics, The University of Chicago, Chicago, Illinois 60637, USA

${ }^{2}$ Department of Chemistry, The James Franck Institute and The Institute for Biophysical Dynamics,

The University of Chicago, Chicago, Illinois 60637, USA

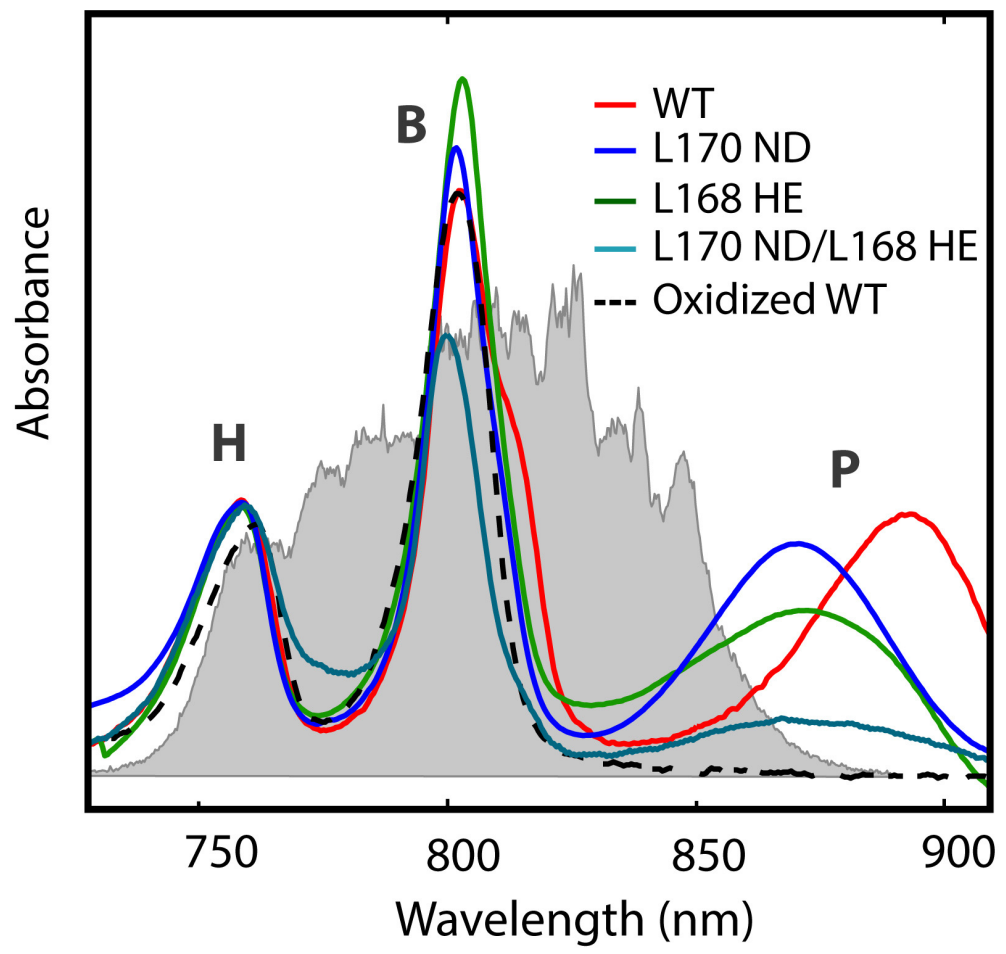

Figure S1- $\boldsymbol{R}$. sphaeroides reaction center absorption spectra and representative narrow laser spectrum. The absorption spectra at $77 \mathrm{~K}$ of the wild type (WT), P-oxidized wild type (Oxidized WT), and three mutants (L170ND, L168HE, L170ND/L168HE) are 


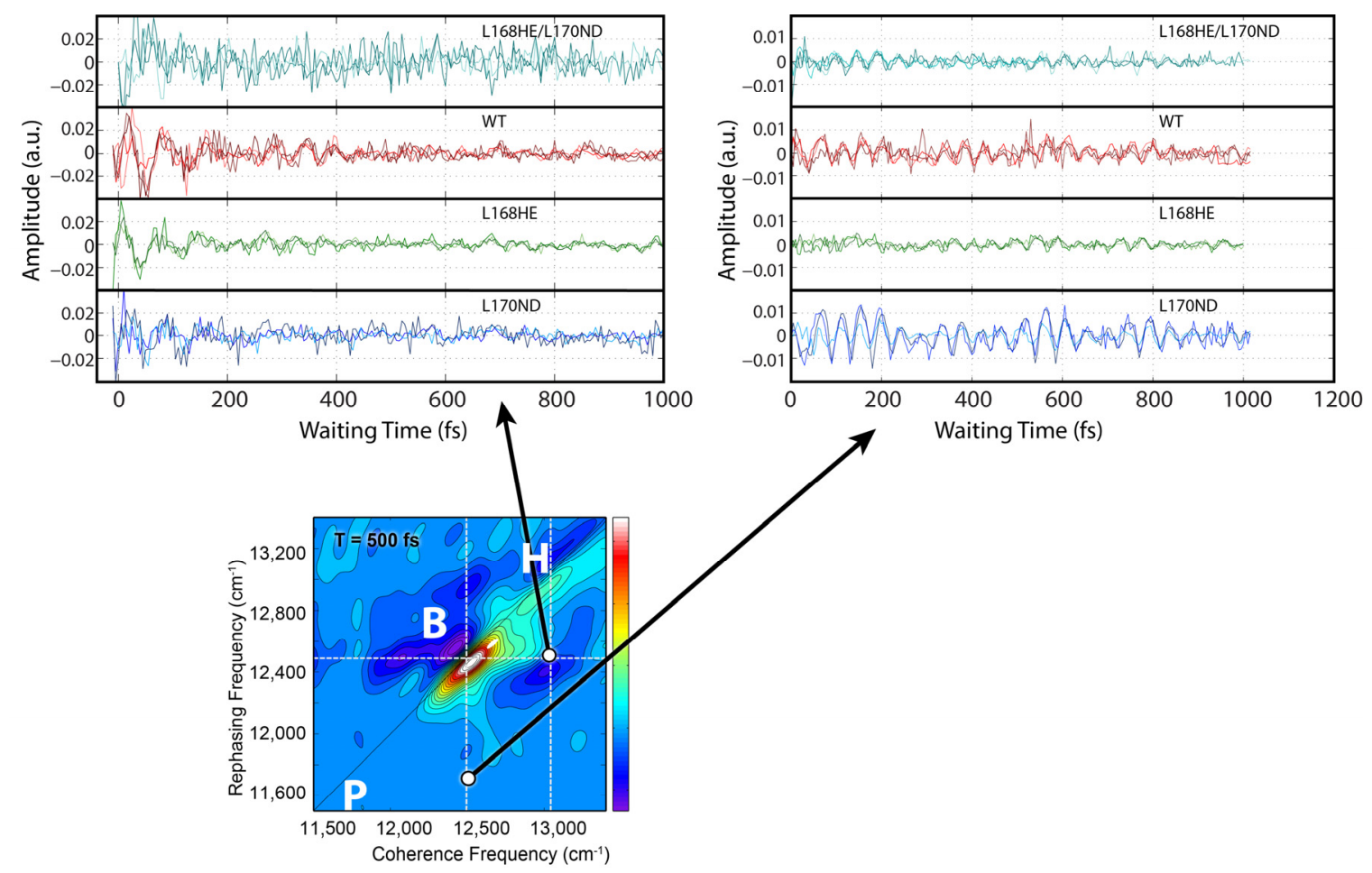

shown. The P-oxidized sample was brought to $\sim 200 \mathrm{mM} \mathrm{K}_{3} \mathrm{Fe}(\mathrm{CN})_{6}$ immediately before freezing.

Figure S2 - Coherences from narrow bandwidth laser spectrum. Traces from the same locations as Figure 2 are shown for each RC sample. Each trace is fit to a double exponential plus offset function and the residuals are shown above. All residual traces are plotted to scale. Multiple traces are shown for each sample. These traces are from separate data sets taken on different days with different pulses. Traces from the $B / H$ crosspeak location correlating to Trace 1 in Figure 2 are shown on the left. Traces correlating to Trace 2 in Figure 2 are shown on the right. This bandwidth did not extend far enough in the red to replicate Trace 3 in Figure 2. 


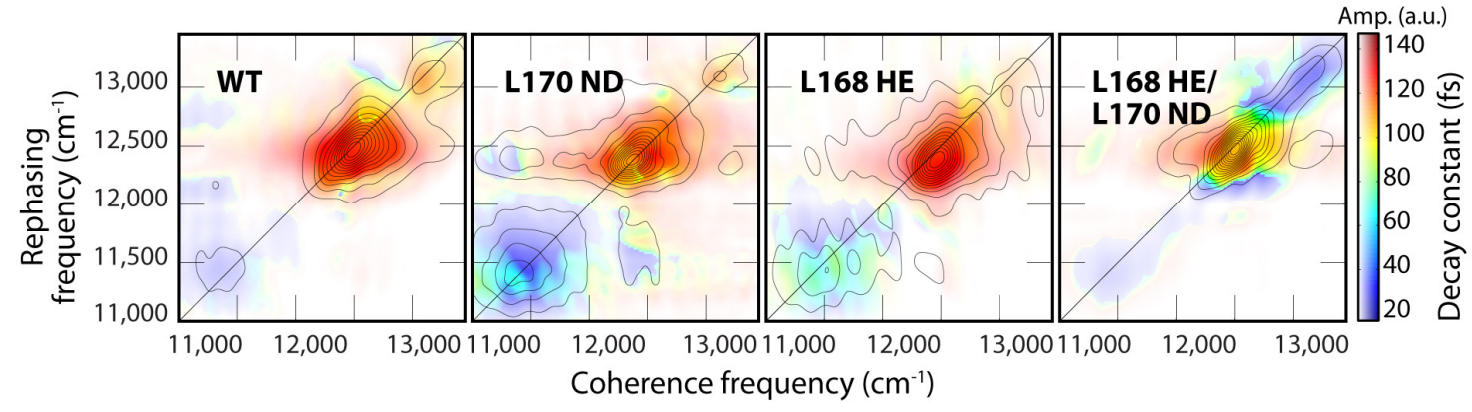

Figure S3 - Decay Associated Spectra for wild type (WT) and mutant RCs. Each set of $2 \mathrm{D}$ data was subject to a biexponential global analysis. The waiting time trace for each pixel is fit to a function of the form $y=A_{1} \exp \left(\frac{T}{130}\right)+A_{2} \exp \left(\frac{T}{1000}\right)+A_{3}$. The resulting parameters are then used as inputs and the rate constants (initially $1 / 130$ and $1 / 1000$ ) are allowed to vary. The result is a short (under $140 \mathrm{fs}$ ) and a longer lifetime (greater than $800 \mathrm{fs}$ ). The shorter lifetime is plotted for each RC sample in this figure. The coloration is determined by the decay constant, while the saturation is determined by the magnitude of the signal.

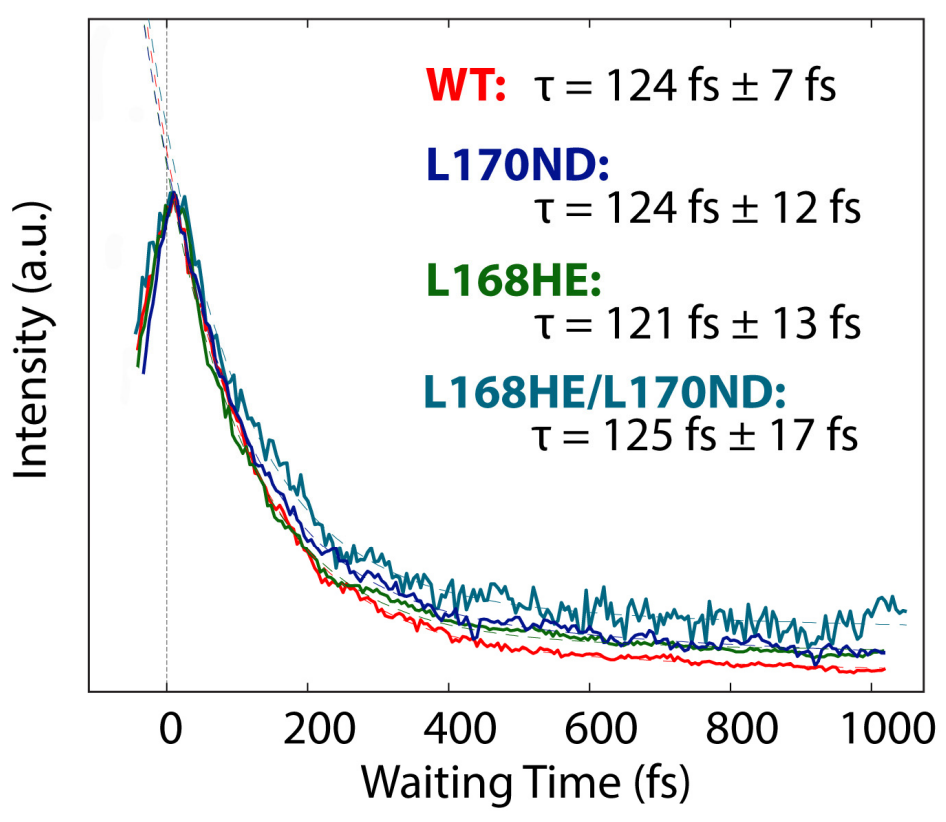

Figure S4-Dynamics of Mutant Reaction Centers with Narrow Laser Spectrum. Waiting time traces of the B peak $\left(\omega_{\tau}=12490 \mathrm{~cm}^{-1}\right.$ and $\left.\omega_{t}=12470 \mathrm{~cm}^{-1}\right)$ for the WT and three mutants. The traces are the average of $n=3$ separate datasets. Biexponential fits are shown as dashed lines for each waiting time trace. The dominant decay constant with one standard deviation is reported for each. The narrow laser spectrum is shown in Figure S1. 


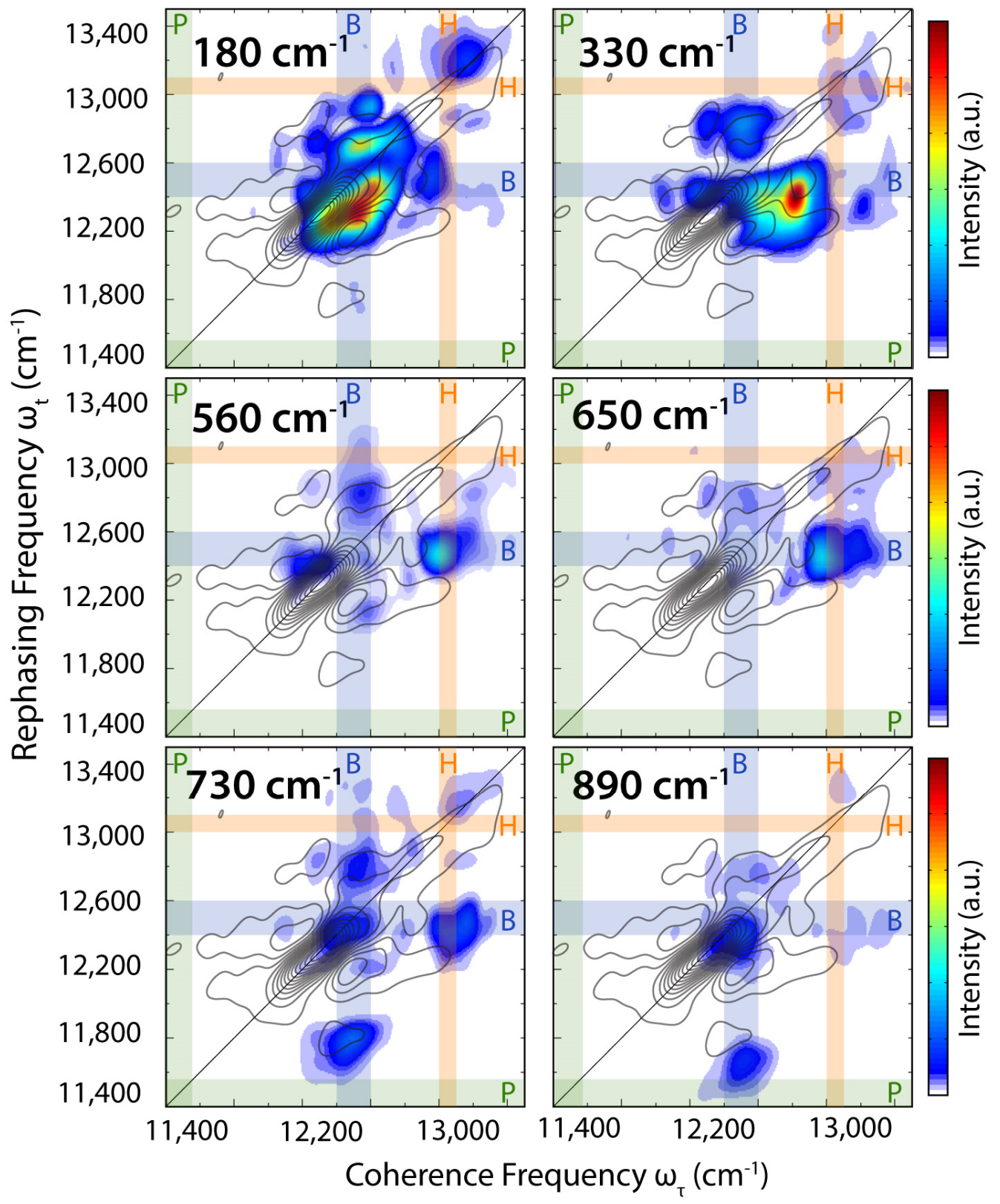

Figure S5-Beat Map for WT Reaction Center using Narrow Laser Bandwidth. The power spectrum amplitudes for the six frequencies in Figure 4a are plotted on the rephasing 2D map for some of the observed modes. The transparent bars highlight energies of the $\mathrm{P}, \mathrm{B}$, and $\mathrm{H}$ exciton energies. The narrow laser spectrum is shown in Figure S1. 


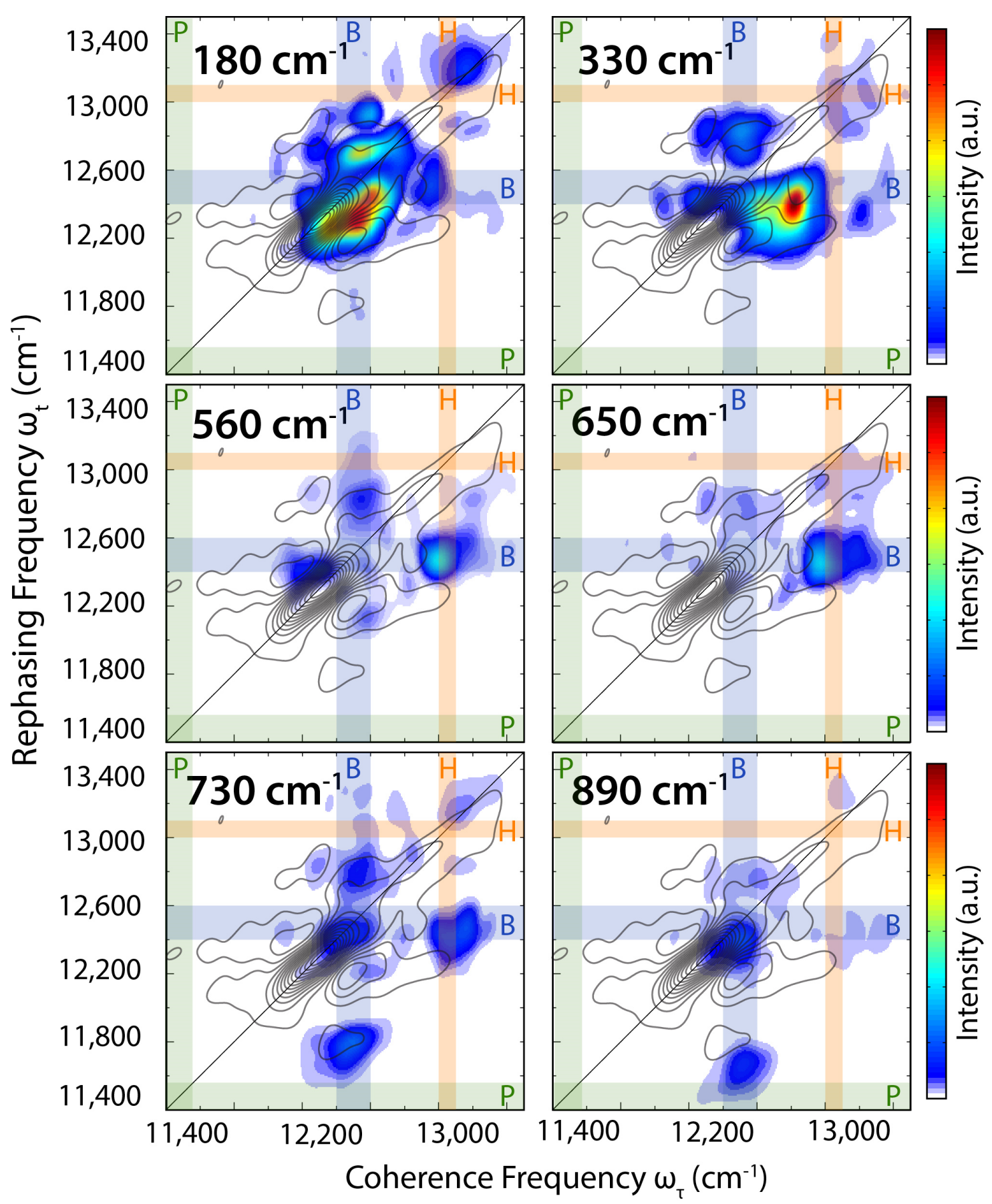

Figure S6 - Amplitude Beat Map for WT Reaction Center with Broadband Pulse. The power spectrum amplitudes for six frequencies in Figure 4a are plotted on the rephasing $2 \mathrm{D}$ map for some of the observed modes. The transparent bars highlight energies of the $\mathrm{P}, \mathrm{B}$, and $\mathrm{H}$ exciton energies. The laser spectrum is shown in Figure 1. 
351802203305607307808901015
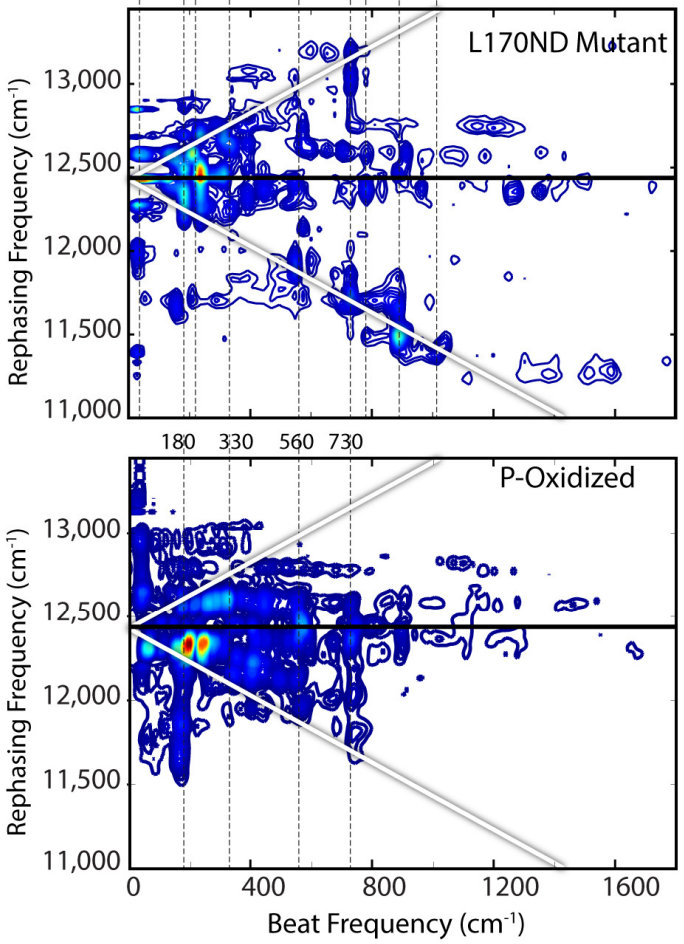

$180220330 \quad 730 \quad 89010401170$
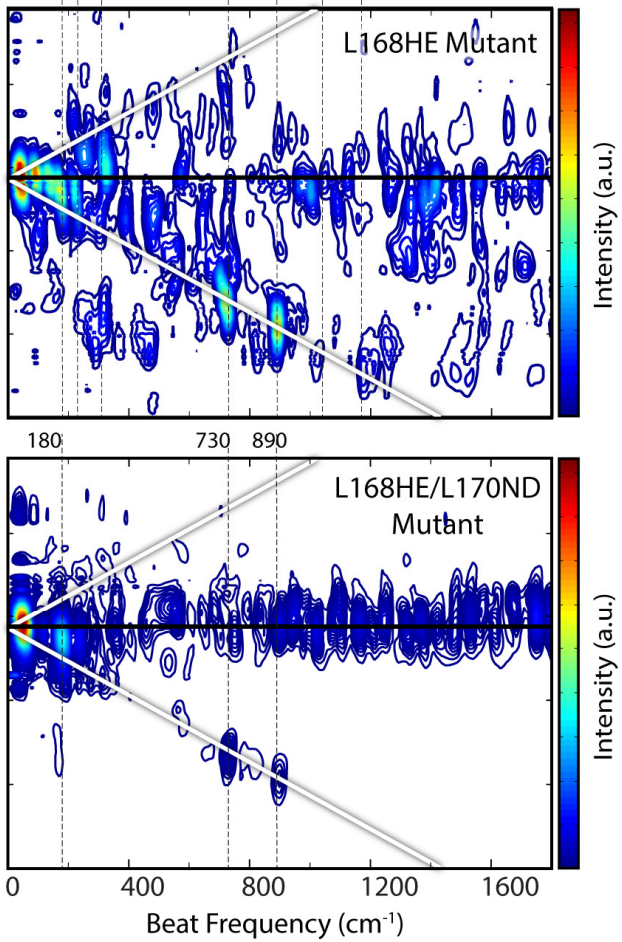

Figure S7- Power spectra of beating in Mutant R. sphaeroides show changes in vibronic coupling with perturbations to $\mathbf{P}$ band transition energy. Power spectrum slices along rephasing frequencies at the $B$ band transitions $\left(\omega_{\tau}=12,490 \mathrm{~cm}^{-1}\right)$ energies for each mutant and the P-oxidized reaction center. The solid black line in each spectrum indicates the energy that the slice was taken, while the white lines represent expected vibrational peak locations. The P-oxidized data was taken with the narrow bandwidth laser, so we can't detect the presence of any features on the red side (bottom) of the plot or vibrations at high energy such as the 1040 or 1170 cm-1 modes. The mutant spectra were all taken with the broad bandwidth. The mutant and P-oxidized RCs have the similar vibrational modes below the B band energy, but the higher frequency modes, 1040 and $1170 \mathrm{~cm}^{-1}$ are weaker or missing, while the $890 \mathrm{~cm}^{-1}$ appears stronger in the power spectra. 


\section{a)}

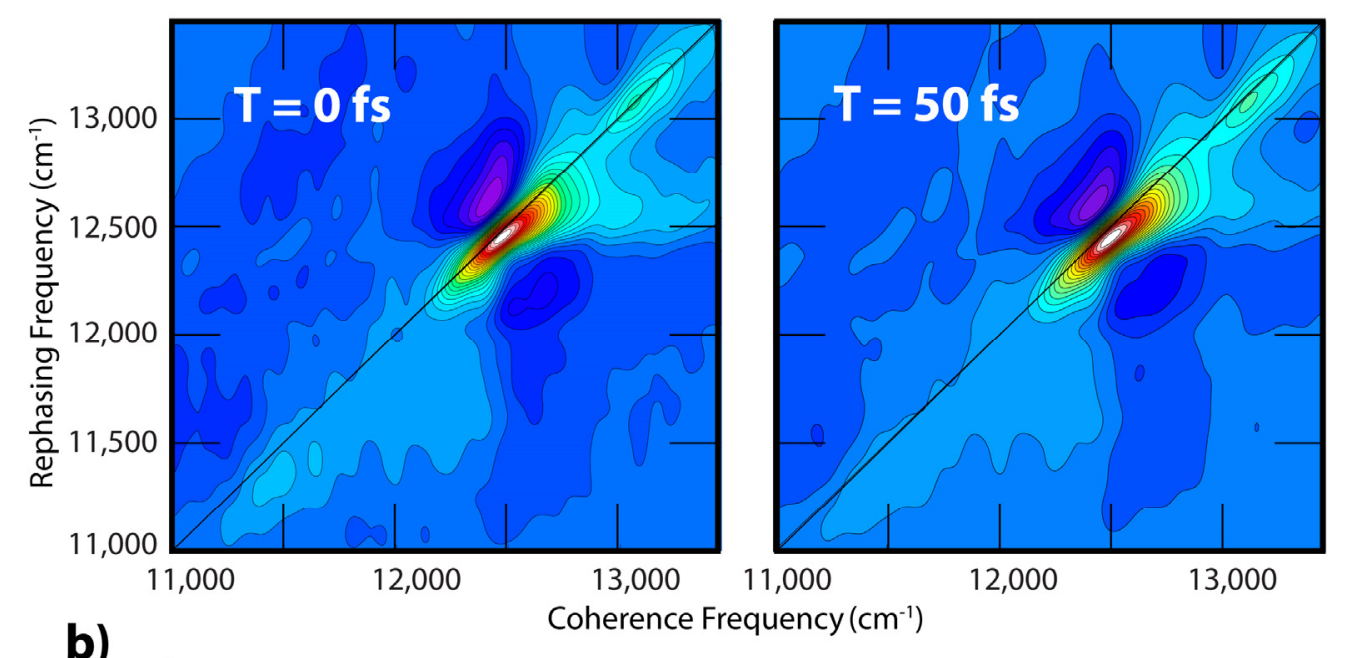

b)

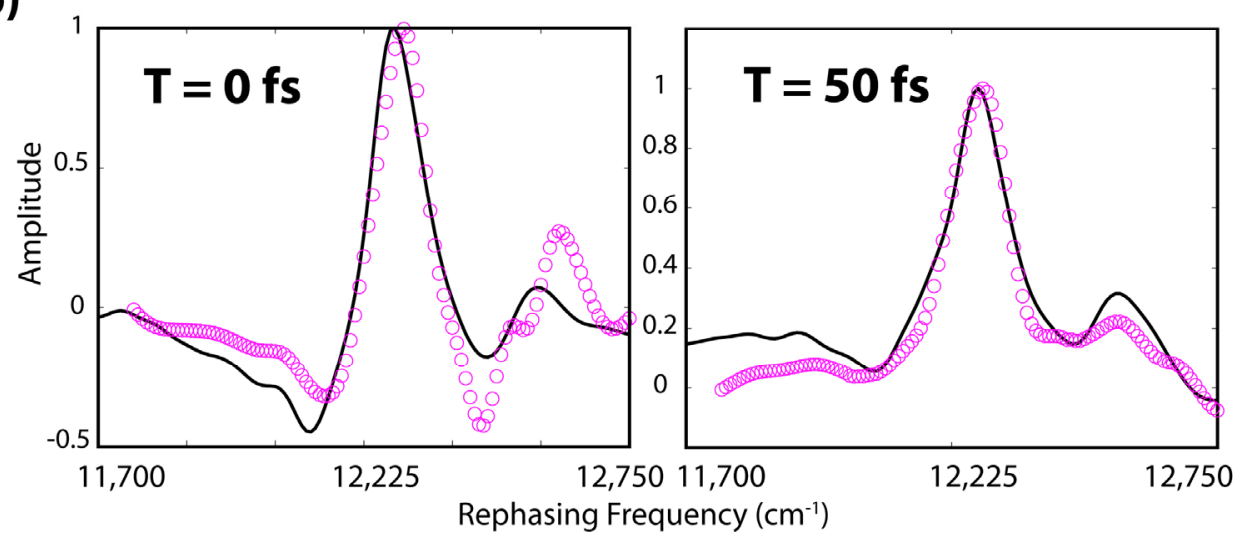

Figure S8- Phasing of Data. a) Phased spectra at waiting times of 0 and $50 \mathrm{fs}$. b) Phasing was constructed by fitting the projection of the 2D spectra on the rephasing axis to separately acquired pump probe data. Pump probe data is shown in magenta open circles. Black lines are the projection of the phased data. A single set of phasing parameters are applied to all waiting times within a data set. 


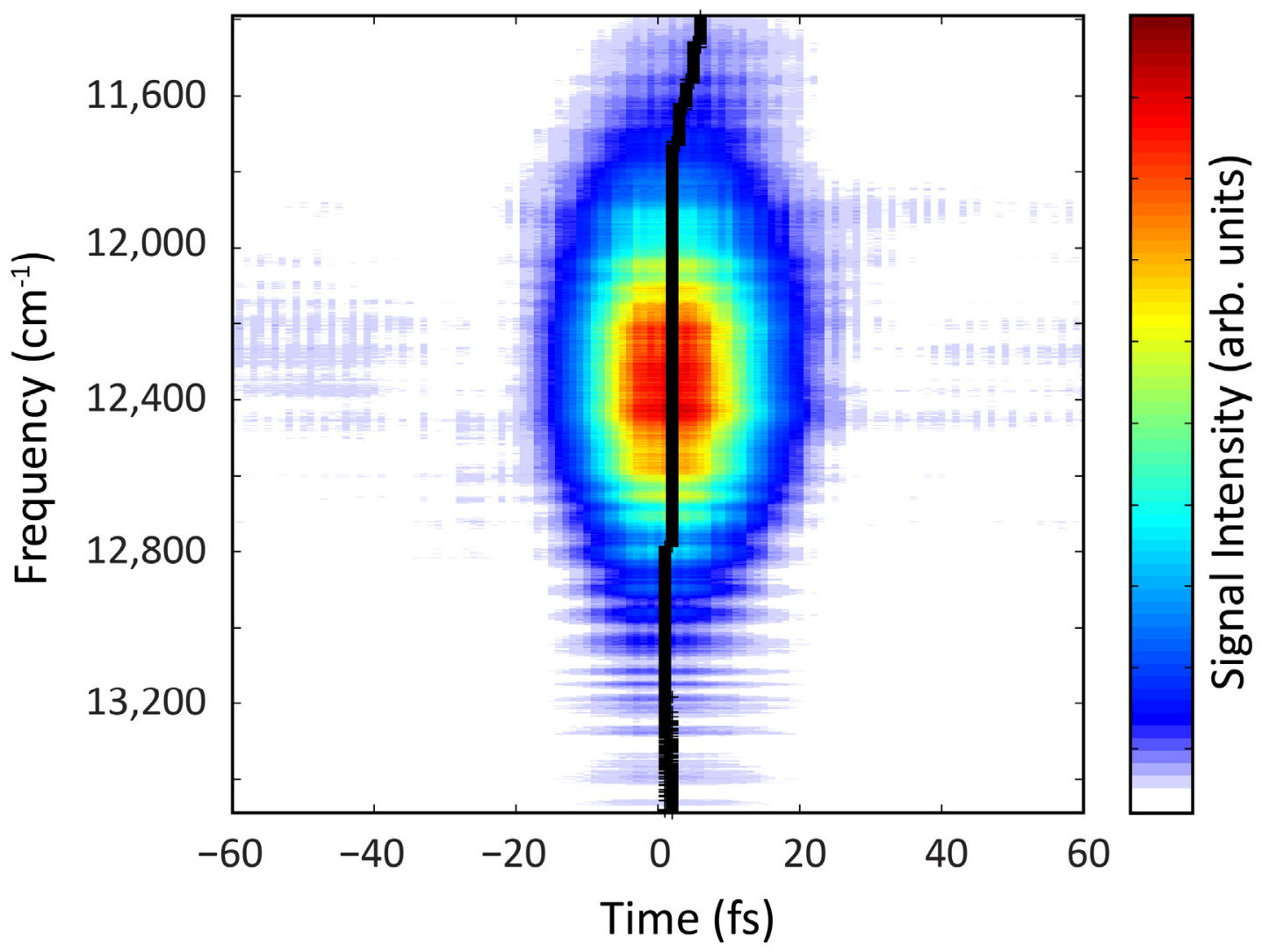

Figure S9- Transient-Grating Frequency-Resolved Optical Grating (TG-FROG) showing a short pulse. Each horizontal slice through time is fit to a Gaussian function and the peak of each Gaussian is marked with a black cross to demonstrate the absence of any significant chirp. 